See discussions, stats, and author profiles for this publication at: https://www.researchgate.net/publication/348484048

\title{
Clay Micromechanics: An Analysis of Elementary Mechanisms of Clay Particle Interactions to Gain Insight into Compression Behaviour of Clay
}

Chapter · January 2021

DOI: 10.1007/978-3-030-64514-4_13

\section{CITATIONS}

7 authors, including:

Angela Casarella

Université Grenoble Alpes

5 PUBLICATIONS O CITATIONS

SEE PROFILE

Alice Di Donna

University of Grenoble

34 PUBLICATIONS 580 CITATIONS

SEE PROFILE
READS

20

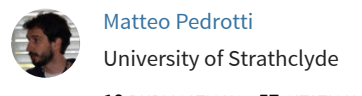

19 PUBLICATIONS 57 CITATIONS

SEE PROFILE

65) Arianna Gea Pagano

University of Strathclyde

7 PUBlications 11 Citations

SEE PROFILE

Some of the authors of this publication are also working on these related projects:

Project Grouting of well leakage and migration pathways: Biogrouting and colloidal silica View project

The Relation between Particle Properties, Flow Microstructure, and Bulk Powder Flow View project 


\title{
Clay Micromechanics: an analysis of elementary mechanisms of clay particle interactions to gain insight into compression behaviour of clay
}

\author{
A. Tarantino ${ }^{1, *}$, A. Casarella ${ }^{2}$, M. Pedrotti ${ }^{1}$, A. Di Donna ${ }^{2}$, A. Pagano ${ }^{1}$, B. de \\ Carvalho Faria Lima Lopes ${ }^{1}$, V. Magnanimo ${ }^{3}$ \\ ${ }^{1}$ Department of Civil and Environmental Engineering, University of Strathclyde (Scotland, UK) \\ "alessandro.tarantino@strath.ac.uk \\ ${ }^{2}$ Univ. Grenoble Alpes, CNRS, Grenoble INP, 3SR, Grenoble (France) \\ ${ }^{3}$ Multiscale Mechanics Group, University of Twente, Enschede (the Netherlands)
}

\begin{abstract}
The macroscopic response of geomaterials is controlled by the processes occurring at the microscale. Understanding these processes is key to interpret experimental data, understand fundamental modes of stress-strain behaviour, inform 'continuum' macroscopic constitutive models, and develop quantitative predictive tools based on Discrete Element Method (DEM) approaches. Unlike granular materials, mechanisms at the particle scale controlling macro-mechanical behaviour of clays are still largely ignored. This paper presents an analysis of elementary mechanisms of clay particle interactions with the aim of gaining an insight into behaviour of clay and advance the process of defining suitable contact laws to be implemented into DEM formulations.
\end{abstract}

Keywords: Clay micro-mechanics, Clay particle interaction, Pore-fluid chemistry, Temperature

\section{Introduction}

The mechanical response of geomaterials is controlled by the interactions between particles. An understanding of such interactions is a critical step in soil mechanics as it allows us to design experiments, interpret experimental data, and understand fundamental mechanisms of behaviour. It also allows us to inform 'continuum' constitutive models and a classic example is represented by the original Cam-Clay model (Roscoe, and Schofield, 1963), the progenitor of the constitutive elasto-plastic models for clays. This 
is based on the assumption that the work input associated with plastic deformation is dissipated entirely in friction according to the following equation

$$
p^{\prime} \delta \varepsilon_{p}^{p}+q \delta \varepsilon_{q}^{p}=M p^{\prime} \delta \varepsilon_{q}^{p}
$$

where $p$ and $q$ are the isotropic and deviator stress respectively, $\varepsilon_{p}^{p}$ and $\varepsilon_{q}^{p}$ are the isotropic and deviator plastic strain respectively, and $M$ is the stress ratio at the critical state. Critical. This model implicitly assumes that clay particles are in contact and slip during shear distortion thus dissipating energy in friction. This mechanism is somehow inconsistent with the idea that clay particle interactions are mainly governed doublelayer repulsion forces, i.e. clay particles 'float' in the pore-fluid similarly to point charges of same sign floating in a dielectric medium.

Pedrotti \& Tarantino (2018) presented experimental evidence that contact establishes between the positively charged edge and the negatively charged face. Particles in edgeto-face configuration are responsible for the open soil skeleton structure and the slippage at the edge-to-face contact generates the mechanism of plastic deformation (Fig. 1).
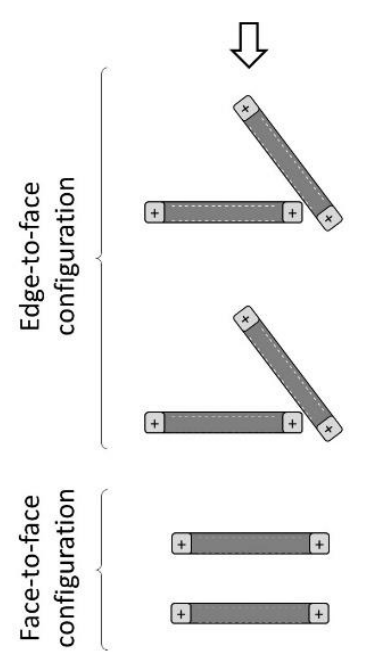
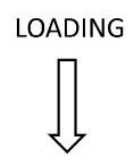

(a)

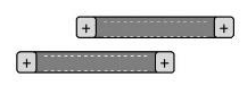

(b)

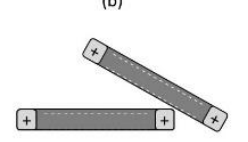

(c)

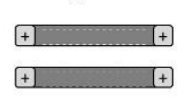

UNLOADING
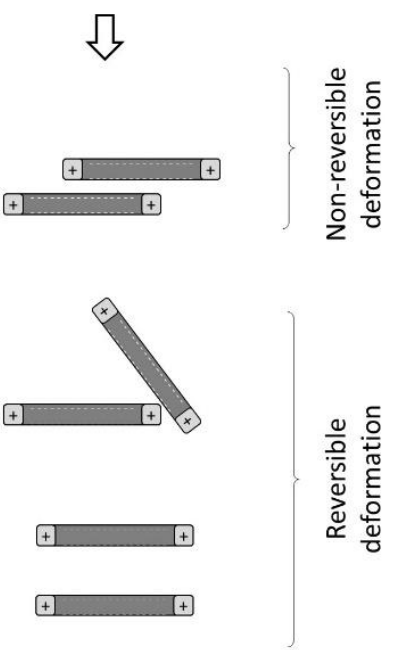

Fig. 1. Mechanisms of plastic and elastic deformation in clays according to Pedrotti \& Tarantino (2018). (a) Plastic deformation associated with slippage at the edge-to-face contact. (b) Elastic response associated with compression of the double-layer in edge-to-face configuration. (c) Elastic response associated with compression of the double-layer in face-to-face configuration.

Based on this concept, Pagano et al. (2020) have presented a Discrete Element Method (DEM) framework where contact laws were designed according to the conceptual model developed by Pedrotti \& Tarantino (2018). Contact laws were successfully tested against their ability to reproduce qualitatively the compression behaviour of clay with porewater of varying $\mathrm{pH}$ and dielectric permittivity. The two-dimensional DEM framework 
was intentionally kept simple in order to demonstrate the robustness of the micromechanical concept underlying the proposed contact laws. DEM modelling should be further advanced in the future to achieve to better satisfactory quantitative prediction of clay behaviour (by considering three-dimensional formulation and refining contact laws).

A DEM model represents potentially a powerful tool to address problems of 'environmental' geotechnics. Thermal effects (e.g. energy geostructures) and pore-chemistry effects (e.g. leachate from landfill) are already embedded in the DEM formulation as electrochemical inter-particle forces depend on temperature and dielectric permittivity/electrolyte concentration of the pore-fluid. As an example, Casarella et al. (2020) have explored conceptually the micromechanical response of clays subjected to temperature changes to address the problem of the design of energy geostructures ( $\mathrm{Di}$ Donna and Laloui, 2013).

This paper presents an analysis of elementary mechanisms of clay particle interactions with the aim of gaining an insight into of clay and advance the process of defining suitable contact laws to be implemented into DEM formulations. The discussion is limited to non-active kaolinite clays (characterised by a 1:1 mineralogical structure) and response of clays in compression.

\section{Surface charges on kaolinite}

\subsection{Traditional model (Pedrotti's model)}

The negative charge of the particle face (surface charge on the basal planes) results from isomorphic substitutions and is generally considered to be a permanently structural charge (Van Olphen, 1977). The charge of the particle edge results from the protonation or deprotonation of hydroxyl groups of edges and it is $\mathrm{pH}$-dependent (the charge of the edge is positive at low $\mathrm{pH}$ values and becomes negative at higher $\mathrm{pH}$ values (Fig. 2). According to Schroth \& Sposito (1997), two values of $\mathrm{pH}$ can be identified, the Point of Zero Net Charge (PZNC) where the positive charge at the particle edge balances the negative charge at the surface ( $\mathrm{PZNC}=3-4$ for kaolinite) and the Point of Zero Net Proton Charge (PZNPC) where the edge assumes zero charge ( $\mathrm{PZNPC}=5-6$ for kaolinite). The Cation Exchange Capacity (CEC), the total capacity of a soil to hold exchangeable cations, can be been used to measure the charge of kaolinite particles.

$$
\sigma=\frac{C E C}{S S A} 10^{-5} N_{A} e
$$

where CEC $[\mathrm{meq} / 100 \mathrm{~g}]$ is the cation exchange capacity, $\mathrm{SSA}[\mathrm{m} 2 / \mathrm{g}]$ is the specific surface area $[\mathrm{m} 2 / \mathrm{g}], N_{\mathrm{A}}$ is the Avogadro number $\left(N_{\mathrm{A}}=6.022 \cdot 10^{23}\right)$, and $e$ is the electron charge $\left(e=1.602 \cdot 10^{-19} \mathrm{C}\right)$. Conceptually, the CEC depends on both the negative charge of the face and the positive/negative charge of the edge. It can be used to measure the 
negative electrical charge of the surface only at the PZNPC. Values of surface charge created by isomorphic substitutions, assumed to generate the negative charge at the surface, have been determined for reference kaolinite clays and are in the range of 50-70 $\mathrm{mC} / \mathrm{m} 2$ (Table 1).

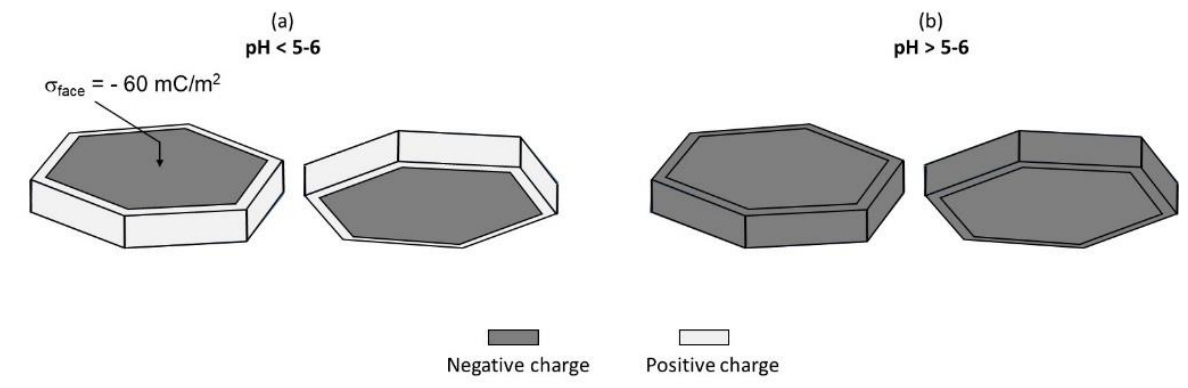

Fig. 2. Pedrotti's model for surface charge on kaolinite particle. (a) acidic pore fluid (low pH). (b) alkaline pore fluid (high $\mathrm{pH}$ ).

Table 1 Surface charge created by isomorphic substitutions

\begin{tabular}{lcccc}
\hline Reference & Name & $\begin{array}{c}\text { SSA } \\
{[\mathrm{m} 2 / \mathrm{g}]}\end{array}$ & $\begin{array}{c}\mathrm{CEC} \\
{[\mathrm{mmol} / 100 \mathrm{~g}]}\end{array}$ & $\begin{array}{c}\sigma \\
{[\mathrm{mC} / \mathrm{m} 2]}\end{array}$ \\
\hline Schroth \& Sposito 1997 & KGa-1 & 8.8 & 0.63 & 69 \\
\hline Schroth \& Sposito 1997 & KGa-2 & 21.6 & 1.36 & 61 \\
\hline Khawmee et al 2013 & KGa-2 & 21 & 1.02 & 47 \\
\hline Bolland et al 1976 & API-9 & 20 & 1.5 & 72 \\
\hline
\end{tabular}

\subsection{Utah model}

The surface charge of the kaolinite face and edges have been measured recently using the Atomic Force Microscope (AFM). An entirely different picture emerged from these measurements (Fig. 3). The alumina (tetrahedral) face is positively charged at low $\mathrm{pH}$ values and negatively charged at high $\mathrm{pH}$ values and the silica (tetrahedral) face is always charged negatively, with values of density charge that become more negative as pH values increase (Gupta \& Miller, 2010; Kumar et al., 2017). On the other hand, the particle edge is always negatively charged and its charge is nearly independent of $\mathrm{pH}$ values $\left(\sigma_{\text {edge }} \cong-10 \mathrm{mC} / \mathrm{m} 2\right)$ (Liu et al. 2014). 

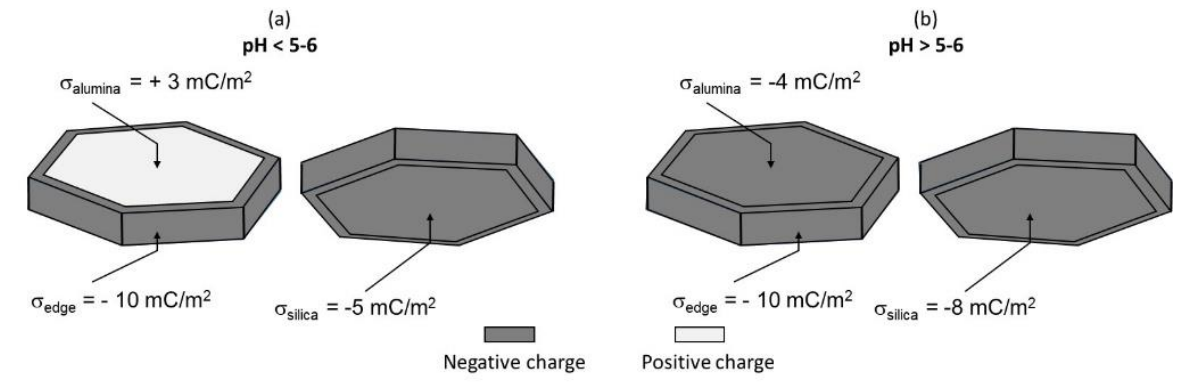

Fig. 3. Utah model for surface charge on kaolinite particle. (a) acidic pore fluid (low pH). (b) alkaline pore fluid (high $\mathrm{pH}$ )

A positively charged alumina face and a negatively charged silica face does not necessarily mean that there in an attraction if two particles have the silica and alumina faces opposite each other.

Values of the charge of the alumina and silica faces are shown in Fig. 4. At $\mathrm{pH}=5$, which is a typical value obtained when mixing Speswhite kaolin with standard deionised water, the net charge is negative (De Carvalho Faria Lima Lopes et al., submitted). If one assumes that the negative and positive charges of the silica and alumina faces respectively are localized at the outer surface $\left(\sigma_{\text {silica }}=-5 \mathrm{mC} / \mathrm{m}^{2}, \sigma_{\text {alumina }}=+3 \mathrm{mC} / \mathrm{m}^{2}\right.$ according to Fig. 4), the electrical potential generated by the two layers outside the particle (calculated for the case of a disk uniformly charged as discussed later in the paper) would always be negative, even in close proximity of the surface. As result, the electrical potential would be negative all around the particle (both faces and edge) and it might be difficult to justify flocculation observed experimentally for Spewshite kaolin at $\mathrm{pH}$ values of 5 . The Utah model in Fig. 3 requires to be corroborated by some further direct and indirect experimental evidence and has been 'parked' at the moment (but it will be further explored in the future). This paper only explores the more traditional Pedrotti's model. Examples are developed by assuming that the negative charge at the surface is characterised by a net value of $\sigma_{\text {net }}=-2 \mathrm{mC} / \mathrm{m}^{2}$ (which is associated with $\mathrm{pH}=5$ according to Fig. 4).

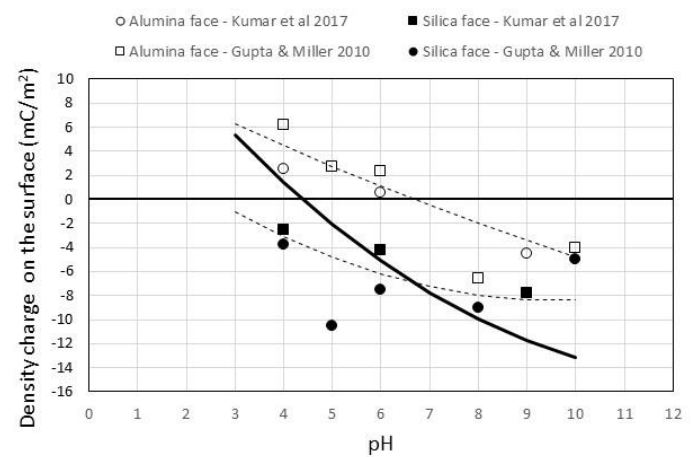

Fig. 4. Measurement of surface density charge via Atomic Force Microscope (De Carvalho Faria Lima Lopes et al., submitted). 


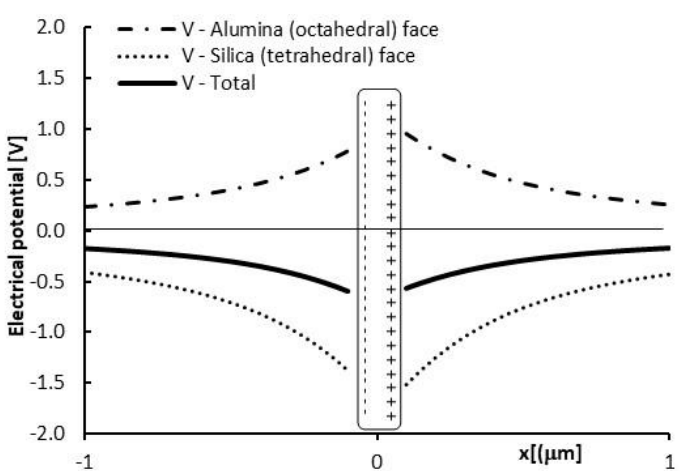

Fig. 5. Electrical potential generated by two adjacent discs of constant charge $(R=0.5 \mathrm{~mm}, t=0.1 \mathrm{~mm}$, $\left.\sigma_{\text {silica }}=-5 \mathrm{mC} / \mathrm{m}^{2}, \sigma_{\text {alumina }}=+3 \mathrm{mC} / \mathrm{m}^{2}\right)$.

\section{Interaction of double-layers generated by two (infinite) plane parallel sheets of constant charge}

The interaction between particles is first addressed by considering the case of particles in parallel configuration. This allows accounting for the effect of the pore-fluid electrolytes by considering the Gouy-Chapman theory for the diffuse double layer. This will serve as a benchmark for the case where the electrolytes are neglected and interaction is only analysed by considering the effect of charge of the particles. The case of "no electrolyte' is convenient when analysing the edge-to-face configuration, which would indeed be tricky if the electrolytes are included and double-layers effect considered.

\subsection{Single flat double layer}

\subsubsection{Solution of governing equation}

The equation of the diffuse-double layers under the assumptions highlighted in Mitchell and Soga (2005) is given by:

$$
\frac{d^{2} \Psi}{d x^{2}}=\frac{2 n_{0} v e}{\varepsilon_{0} \epsilon_{r}} \sinh \left(\frac{v e \Psi}{k T}\right)
$$

where $\Psi$ is the electrical potential, $x$ the distance from the surface, $n_{0}[1 / \mathrm{mol}]$ the concentration of electrolytes at zero potential (i.e. at $x=\infty$ ), $v$ is the valence, $e$ is the electron charge, $\varepsilon_{0}$ is the dielectric permittivity in vacuum, $\varepsilon_{\mathrm{r}}$ is the relative dielectric permittivity, $k$ is the Boltzmann constant, and $T$ is the absolute temperature. Values of the parameters in Eq. (3) are given in Table 2.

This equation is solved here for a given surface charge $\sigma$, i.e. for the following boundary conditions: 


$$
\begin{gathered}
\sigma=-\int_{0}^{\infty} \rho d x=\varepsilon \int_{0}^{\infty} \frac{d^{2} \Psi}{d x^{2}} d x=-\varepsilon\left(\frac{d \Psi}{d x}\right)_{0} \\
\frac{d \Psi}{d x}=0 \text { for } x \rightarrow \infty(\Psi \rightarrow 0)
\end{gathered}
$$

By assuming

$$
y=\frac{v e \Psi}{k T} ; z=\frac{v e \Psi_{0}}{k T} ; K^{2}=\frac{2 n_{0} v^{2} e^{2}}{\epsilon_{0} \varepsilon_{r} k T} ; \xi=K x
$$

where $\Psi_{0}$ is the potential at the surface, Eq. (3) can be written in non-dimensional form

$$
\frac{d^{2} y}{d \xi^{2}}=\sinh y
$$

The solution of the single double-layer equation in non-dimensional form can be derived as follows (van Olphen,1977):

$$
\exp \left(\frac{y}{2}\right)=\frac{\exp \left(\frac{Z}{2}\right)+1+\left[\exp \left(\frac{Z}{2}\right)-1\right] \exp (-\xi)}{\exp \left(\frac{Z}{2}\right)+1-\left[\exp \left(\frac{Z}{2}\right)-1\right] \exp (-\xi)}
$$

This solution is based on the value of the non-dimensional potential at the surface $z$, which then needs to be expressed as a function of the surface charge $\sigma$.

Since

$$
\sigma=-\varepsilon\left(\frac{d \Psi}{d x}\right)_{0}=-\varepsilon \frac{k T}{v e} K\left(\frac{d y}{d \xi}\right)_{0}=\varepsilon \frac{k T}{v e} 2 \sinh \left(\frac{z}{2}\right)
$$

then

$$
\Psi_{0}=\frac{2 k T}{v e} \ln \left[\frac{\sigma}{\left(8 n_{0} \epsilon_{0} \varepsilon_{r} k T\right)^{\frac{1}{2}}}+\sqrt{\frac{\sigma^{2}}{8 n_{0} \epsilon_{0} \varepsilon_{r} k T}+1}\right]
$$

The potential at the surface $\Psi_{0}$ can be therefore derived from the surface charge $\sigma$ using Eq. (9). In turn, this allows for the computation of $z$ from Eq. (5), which is the boundary condition required to derive the potential in non-dimensional form via Eq. (7).

Table 2. Diffuse-double layer parameters

\begin{tabular}{lcccc}
\hline$k\left[\mathrm{~J} \mathrm{~K}^{-1}\right]$ & $e[\mathrm{C}]$ & $\mathrm{N}_{\mathrm{A}}\left[\mathrm{mol}^{-1}\right]$ & $\varepsilon_{0}\left[\mathrm{C}^{2} \mathrm{~J}^{-1} \mathrm{~m}^{-1}\right]$ & $v[-]$ \\
\hline $1.38 \cdot 10^{-23}$ & $1.602 \cdot 10^{-19}$ & $6.022 \cdot 10^{23}$ & $8.8542 \cdot 10^{-12}$ & 1 \\
\hline
\end{tabular}




\subsubsection{Effect of pore-chemistry and temperature}

The distributions of the electrical potential for a kaolinite particle with surface charges of $\sigma=2 \mathrm{mC} / \mathrm{m}^{2}$ and $\sigma=60 \mathrm{mC} / \mathrm{m}^{2}$ are shown in Fig. 6. A concentration of $C_{0}=2 \cdot 10^{-5} \mathrm{M}$ was assumed in the calculations according to the value estimated by Pedrotti \& Tarantino (2018) for Spewshite kaolin mixed with deionised water $\left(n_{0}=10^{3} \times N_{\mathrm{A}} \times C_{0}\right)$.

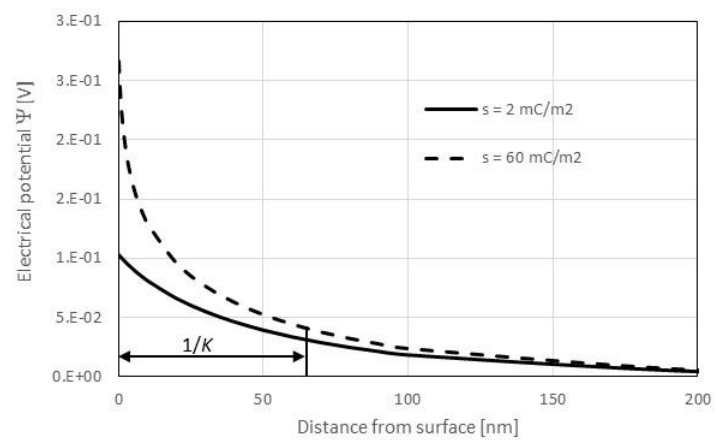

Fig. 6. Electrical potential generated by single flat double layer. $\left(\sigma=2 \mathrm{mC} / \mathrm{m}^{2}\right.$ or $\sigma=60 \mathrm{mC} / \mathrm{m}^{2}, T=293 \mathrm{~K}$, $\left.C_{0}=2 \cdot 10^{-5} \mathrm{M}, \varepsilon_{\mathrm{r}}=80\right)$.

The electrical potential decreases from the surface and the extension of the double-layer can be measured by its thickness, $1 / K$ (Mitchell and Soga, 2005)

$$
\frac{1}{K}=\sqrt{\frac{\epsilon_{0} \varepsilon_{r} k T}{2 n_{0} v^{2} e^{2}}}
$$

which is equal to $68 \mathrm{~nm}$ in this case. The effect of dielectric permittivity, concentration, and temperature on the thickness of the double layer is shown in Fig. 7. The qualitative trend for $\varepsilon_{\mathrm{r}}$ and $C_{0}$ can be derived straightforwardly from Eq. (10). The effect of temperature is less intuitive because one would expect the thickness $1 / K$ to increase with $T$ according to Eq. (10). However, the dielectric permittivity also decreases with temperature and these two effects nearly balance each other.
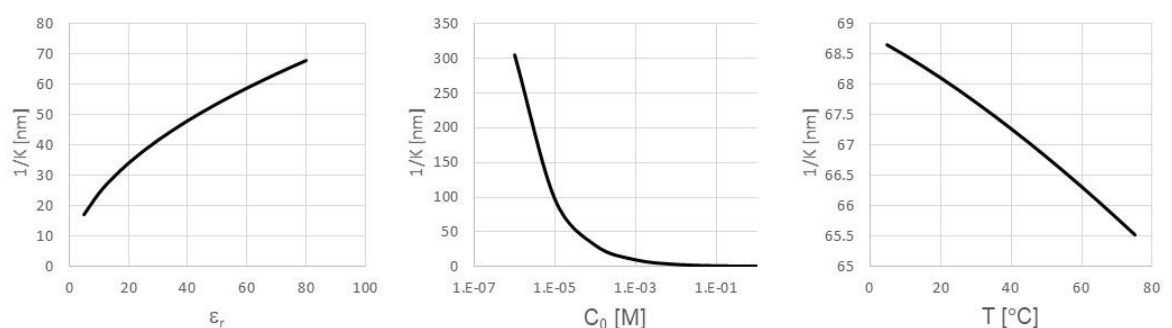

Fig. 7. Effect of temperature (a), dielectric permittivity (b), and ion concentration (c) on the 'thickness' of the double-layer (reference values $\sigma=2 \mathrm{mC} / \mathrm{m}^{2}, T=293 \mathrm{~K}, C_{0}=2 \cdot 10^{-5} \mathrm{M}, \varepsilon_{\mathrm{r}}=80$ ). 
It is interesting to separate these two effects on the entire electrical potential distribution as shown in Fig. 8 (the dielectric permittivity at $\mathrm{T}=20^{\circ} \mathrm{C}$ and $\mathrm{T}=80^{\circ} \mathrm{C}$ are $\varepsilon_{\mathrm{r}}=80$ and $\varepsilon_{\mathrm{r}}=61$ respectively). The dashed curve represents the combined effect of temperature increase and dielectric permittivity decrease and shows that the electrical potential increases overall, at least for the surface charge and electrolyte concentration considered in this example.

The resulting electrical potential distribution combines the direct effect of temperature (causing an increase in electrical potential as shown by the dotted line) and the effect of dielectric permittivity (causing a decrease increase in electrical potential far from the surface as shown by the thin continuous line).

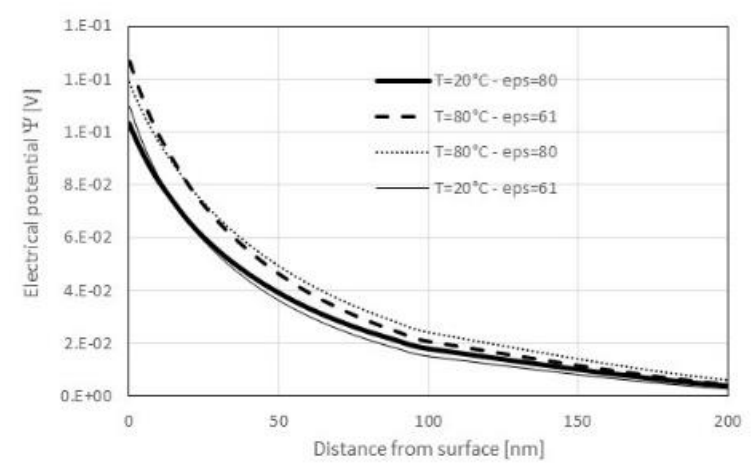

Fig. 8. Combined effect of temperature and dielectric permittivity on electrical potential $\left(\sigma=2 \mathrm{mC} / \mathrm{m}^{2}\right.$, $T=293 \mathrm{~K}$ or $\mathrm{T}=353 \mathrm{~K}, C_{0}=2 \cdot 10^{-5} \mathrm{M}, \varepsilon_{\mathrm{r}}=80$ ).

\subsection{Interacting flat double layers (at constant charge)}

\subsubsection{Solution of governing equation}

For the case of interacting double layers, i.e. two flat particles opposite each other at a half-distance $d$, Eq. (3) is solved for the following boundary conditions:

$$
\begin{gathered}
\sigma=-\int_{0}^{d} \rho d x=\varepsilon \int_{0}^{d} \frac{d^{2} \Psi}{d x^{2}} d x=-\varepsilon\left(\frac{d \Psi}{d x}\right)_{0} \\
\left(\frac{d \Psi}{d x}\right)_{x=d}=0
\end{gathered}
$$

Again the problem is solved here for constant surface charge. According to van Olphen (1977), Eq. (3) cannot be solved analytically. For the numerical solution, the midway non-dimensional potential $u$ at the half-distance $d$ is first determined numerically from the following equality (van Olphen, 1977) 


$$
\int_{z}^{u} \frac{d y}{\sqrt{2 \cosh y-2 \cosh u}}=-K d
$$

with

$$
z=\operatorname{acosh}\left(\frac{\sigma^{2}}{\epsilon_{0} \varepsilon_{r} 4 n_{0} k T}+\cosh u\right)
$$

to account for the condition that the potential at the surface is controlled by the charge. The unknown $u$ appears in both the integrand function and the integral bound and is determined numerically.

The relationship between $u$ and $K \times d$ is shown in Fig. 9a for different values of surface density charge. These relationships differ from Table IX in Verwey \& Overbeek (1948) because the latter is derived for given potential (and not for given constant charge).

Once the midway non-dimensional potential $u$ is determined, the non-dimensional potential $y$ at and distance $x$ can be extracted by the following equality:

$$
\int_{z}^{y} \frac{d y}{\sqrt{2 \cosh y-2 \cosh u}}=-K x
$$

An example of the distribution of the electrical potential is shown Fig. $9 \mathrm{~b}$ for $d=100 \mathrm{~nm}$ and $\sigma=2 \mathrm{mC} / \mathrm{m}^{2}$.
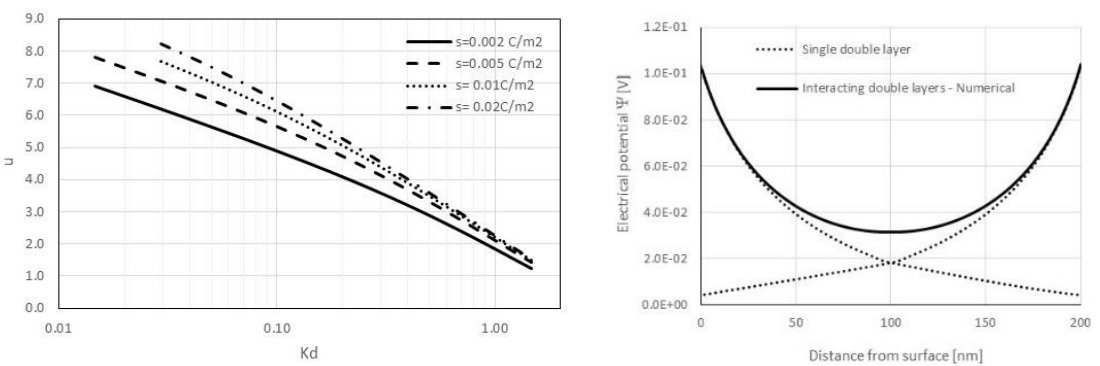

Fig. 9. (a) Relationship between non-dimensional mid-plane electrical potential $u$ and non-dimensional half-distance $K d$ at different values of constant surface density charge. (b) Interacting double layers (reference values $\sigma=2 \mathrm{mC} / \mathrm{m}^{2}, T=293 \mathrm{~K}, C_{0}=2 \cdot 10^{-5} \mathrm{M}, \varepsilon_{\mathrm{r}}=80$ ).

\subsubsection{Interaction pressure}

Traditionally, the interaction pressure between two flat double layers is derived directly from the ionic concentration midway between the plates, which is determined by the value of $u$. The repulsive force is calculated from the osmotic pressure midway between the plates with respect to that of the equilibrium solution. Since these osmotic pressures are determined by the ion concentration, the force is found directly from the excess concentration midway between the plates (van Olphen, 1977).

This approach is questioned here. Eq. (3) is written for the case of infinite sheet and the concentration of the equilibrium solution does not exist in the mathematical model. 
Strictly speaking, the concentration $n_{0}$ does not represent the concentration at infinite distance but just the concentration that would occur at zero potential $\Psi$.

The interaction pressure was calculated here using the classic principle of virtual work used in electrostatics, i.e. the interaction force is derived by differentiating the electrostatic energy (with respect to half-distance $d$ ). Once the distribution of the electrical potential $\Psi$ was derived numerically, the electrostatic energy per unit area $U$ was computed as follows:

$$
U=2\left[\frac{1}{2} \int_{0}^{d} \rho \Psi d x+\frac{1}{2} \sigma \Psi_{0}\right]
$$

where $\rho$ is the volume density charge. This can be expressed as a function of the electrical potential $\Psi$ by considering the Poisson's equation for electrostatics:

$$
U=2\left[\frac{1}{2} \int_{0}^{d}\left(-\epsilon_{0} \varepsilon_{r} \frac{d^{2} \Psi}{d x^{2}}\right) \Psi d x+\frac{1}{2} \sigma \Psi_{0}\right]
$$

The interaction pressure $p$ is then derived by considering

$$
p=\frac{\mathrm{d} U}{\mathrm{~d} d}
$$

The interaction pressure is shown in Fig. 10 for three different values of reference concentration $C_{0}$. It appears that the interaction pressure is bounded by the same value regardless of the concentration. This is not surprising if one considers the case of charged disks with no electrolytes (discussed in the next section). In th9is case, the interaction pressure depends on the size of the disk but the curves all converge to the value of the plane infinite sheet:

$$
p_{p s}=\frac{\sigma^{2}}{2 \epsilon_{0} \varepsilon_{r}}
$$

In other words, there is a finite value of pressure that double layers (plane sheet in presence of electrolytes) or the plane sheets alone (in absence of electrolytes) can sustain.

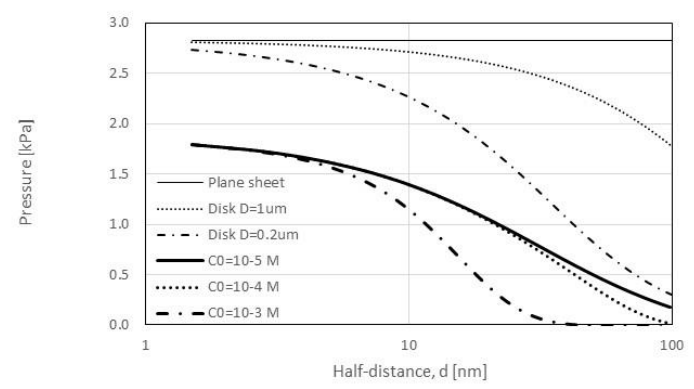

Fig. 10. Interaction pressure versus half-distance between two flat double layers for $C_{0}=10^{-3}, 10^{-4}, 10^{-5} \mathrm{M}$ (reference values $\sigma=2 \mathrm{mC} / \mathrm{m}^{2}, T=293 \mathrm{~K}, \varepsilon_{\mathrm{r}}=80$ ) and comparison with disks and plane sheet with no electrolytes. 
Fig. 11 shows the effect of the surface density charge on the interaction pressure. As for the plane sheet in the absence of electrolytes, the interaction pressure for the double layers increase with the square of the charge. Finally, the effect of temperature is shown in Fig. 12. It can be noticed that the increase in interaction pressure is consistent with the increase in electric potential observed in the single double layer in Fig. 8.

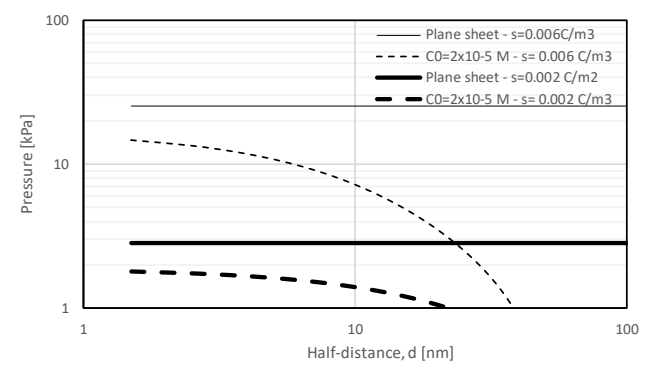

Fig. 11. Effect of surface charge on interaction pressure of double-layers and plane sheets. (reference values $\sigma=2$ or $6 \mathrm{mC} / \mathrm{m}^{2}, T=293 \mathrm{~K}, C_{0}=2 \cdot 10^{-5} \mathrm{M}, \varepsilon_{\mathrm{r}}=80$ ).

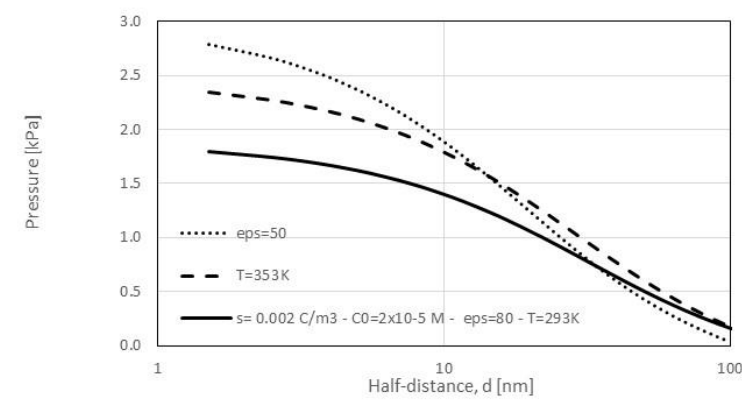

Fig. 12. Effect of temperature and dielectric permittivity on interaction pressure of double-layers (reference values $\sigma=2 \mathrm{mC} / \mathrm{m}^{2}, T=293 \mathrm{~K}, C_{0}=2 \cdot 10^{-5} \mathrm{M}, \varepsilon_{\mathrm{r}}=80$ ).

\section{Interaction of square particles in absence of electrolytes}

\subsection{Face-to-face interaction}

The interaction pressure was analysed for square particles of side $L$ in parallel configuration without considering the presence of electrolytes. he electrostatic energy was calculated as 


$$
U=2\left[\frac{1}{2} \int_{\Omega} \sigma \Psi d \Omega\right]
$$

where $\Psi$ is the electric potential generated on one particle by the adjacent one. The interaction force was then calculated by differentiating the electrostatic energy with respect to the inter-particle spacing as per Eq. (23).

For the case of face to face interaction, the electrical potential was calculated only on the particle axis and assumed to be uniform across the square particle (the error is negligible for inter-particle distances smaller than the size of the square). According to Fig. 13 , the potential is given by

$$
\Psi=\iint_{\Omega} d \Psi=\iint_{\Omega} \frac{1}{4 \pi \epsilon_{0} \varepsilon_{r}} \frac{\sigma z}{\sqrt{x^{2}+y^{2}+z^{2}}} d x d y
$$

For comparison, the interaction pressure was also calculated for the inscribed and circumscribed disk. The electrical potential of a disk on its axis of symmetry is given by:

$$
\Psi=\frac{\sigma}{2 \epsilon_{0} \varepsilon_{r}}\left(\sqrt{a^{2}+R^{2}}-a\right)
$$

where $R$ is the particle radius and $a$ is the inter-particle distance. This leads to the following equation for the interaction pressure according to Eq. (27)

$$
p=\frac{\sigma^{2}}{2 \epsilon_{0} \varepsilon_{r}}\left[\frac{2 a}{2 \sqrt{a^{2}+R^{2}}}-1\right]
$$

For $R \rightarrow \infty$, the equation for the plane sheet shown in Eq. (25) is recovered. The interaction pressure between square particles is compared with the inscribed and circumscribed paticles in Fig. 14. When the spacing tends to zero, the pressures converge to the value of the plane sheet.

It is worth noticing that a charge of $\sigma=2 \mathrm{mC} / \mathrm{m}^{2}$ and a dielectric permittivity of $\boldsymbol{\varepsilon}_{\mathrm{r}}=80$ (water) returns a maximum pressure of about $3 \mathrm{kPa}$, i.e. it would be sufficient to apply a pressure greater than just $3 \mathrm{kPa}$ to cause the particles to stuck together.

If the charge was $\sigma=60 \mathrm{mC} / \mathrm{m}^{2}$ (30 times higher), the limiting repulsive pressure would be 900 times higher since the limiting pressure is proportional to the square of the surface density. As a result, an external pressure of $2700 \mathrm{kPa}$ would be required to cause the particles to stuck together. 


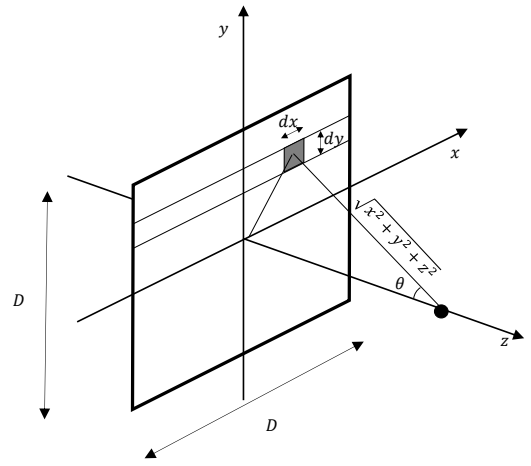

Fig. 13. Computation of the electrical potential on the square axis.

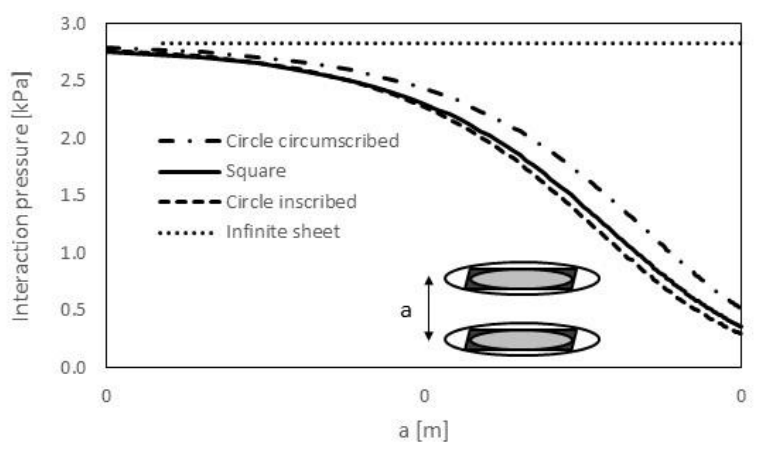

Fig. 14. Interaction pressure in face-to-face configuration $\left(\sigma_{\text {face }}=-2 \mathrm{mC} / \mathrm{m}^{2}, \varepsilon_{\mathrm{r}}=80, L=1 \mu \mathrm{m}\right)$

\subsection{Edge-to-face interaction}

The calculation of the electrical potential for square particle in edge-to-face configuration requires four integrations. As shown in Fig. 15, the electrical potential on a point $\mathrm{P}$ located on the vertical square requires an integration along $x$ and $y$ on the horizontal square. To derive the electrical potential on the vertical square, the electrical potential on the point $\mathrm{P}$ should be integrated along $y$ and $z$ on the vertical square.

Once the potential generated by a square uniformly charged is computed, the case of square particles with different charges on the face and the edge can be analysed via superposition as shown in Fig. 16. 


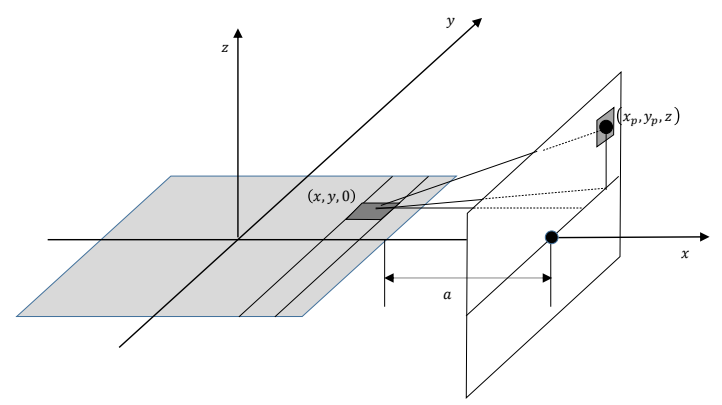

Fig. 15. Computation of the electrical potential on a square vertical particle generated by a horizontal square particle

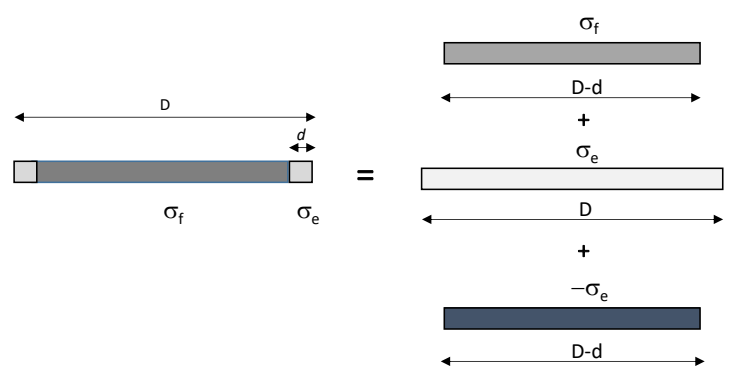

Fig. 16. Superposition to simulate positively charged edge and negatively charged face.

Fig. 17 shows the interaction pressure for the case of i) particles with negative charge on face and edge (high $\mathrm{pH})$, ii) particles with negative charge on the face and positive charge on the edge (low $\mathrm{pH}$ ) witt $\sigma_{\mathrm{e}}=-\sigma_{\mathrm{f}}$, ii) particles with negative charge on the face and positive charge on the edge (low $\mathrm{pH})$ with $\sigma_{\mathrm{e}}=-5 \sigma_{\mathrm{f}}$. As the charge at the edge increases from negative to positive, the repulsion pressure decreases but always remains positive. In other words, the positive charge at the edge does not create an attraction, just depletes the repulsion and depletes the maximum repulsion force that that the edgeto-face contact can sustain.

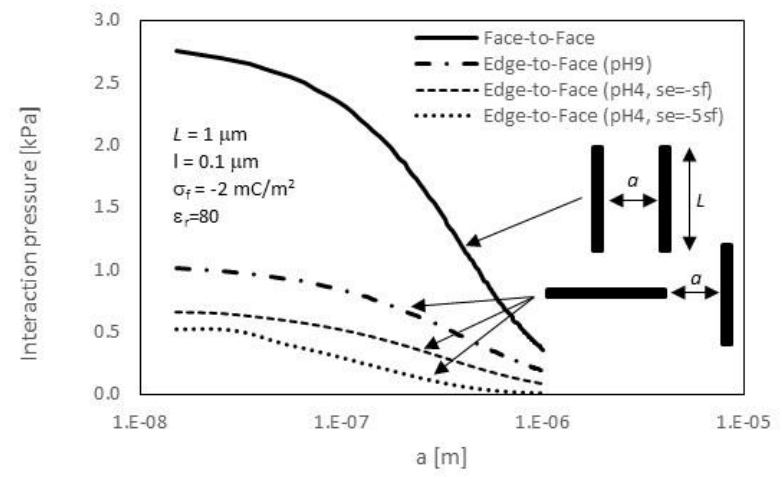

Fig. 17. Edge-to-face interaction compared with face-to-face interaction $\left(\sigma_{\text {face }}=-2 \mathrm{mC} / \mathrm{m}^{2}, \varepsilon_{\mathrm{r}}=80\right)$ 


\section{Discussion}

\subsection{Face-to-face interaction}

The repulsion pressure between two particles in parallel configuration appears to be a finite value (Fig. 10 and Fig. 14). This would imply that particles will get in contact as long as the external pressure exceeds the maximum repulsion pressure that can be deployed by the overlapping electrical fields.

As a result, there is no need to invoke Van der Waals short-range attraction to justify the contact between two particles facing each other. The van der Waals forces are not necessarily responsible for the particles to get in contact, it is sufficient that the external pressure overcomes the limiting one. However, van der Waals forces would prevent the particles to detach once the external pressure is relieved.

This is an irreversible mechanism that can explain some of the irreversible deformation observed in clays as discussed by De Carvalho Faria Lima Lopes et al. (submitted). Pedrotti and Tarantino (2018) made the case that irreversible deformation in compression is associated with the (Fig. 1). However, significant plastic deformation was also observed on clay samples prepared at high $\mathrm{pH}$ values, which generate particles negatively charged all around with no edge-to-face contacts that can establish. This plastic deformation cannot therefore be associated with the disengagement of the edge-to-face inter-particle contact.

The order of magnitude of the maximum repulsion pressure is represented by the pressure between two (infinite) plane sheets, and this is proportional to the square of the surface charge density and inversely proportional to the dielectric permittivity.

When moving from water to air, the dielectric permittivity decreases by 80 times and the maximum sustainable external pressure would then increase by 80 times. It is then much more difficult to 'attach' particles when compressing dry kaolinite powder than kaolinite prepared from slurry. As a result, the component of irreversible compression due to particles getting in contact and then 'bonded' by the van der Waals forced should be more pronounced as the dielectric permittivity increases.

The electrolyte concentration affects the double layer interaction but not the limiting repulsion pressure as shown in Fig. 10. This can partly explain why Wang \& Siu (2006) and Wahid et al. (2011) found that compression behaviour of kaolinite is not affected by pore fluid electrolyte concentration and why Chen et al. (2000) noted that the change in compressibility due to electrolyte adjunction was noticeable only for overburden stress lower than 300 $\mathrm{kPa}$ (consistently with the double layer interaction). 


\subsection{Edge-to-face interaction}

The positively charged edge of a particle is not necessarily attracted by the negatively charged face to create an edge-to-face contact. As shown in Fig. 17, the interaction between two orthogonal particles is dominated by the net negative charge and the overall interaction remains repulsive even if the edge is positively charged. In other words, the particles will not get in contact if there is not an external force pushing them against each other.

However, the positive charge of the edge depletes the force required to establish contact making it very small. Brownian motions might therefore be sufficient to generate edgeto-face attraction for the case where the edge is positive. The edge-to-face contacts are generated more easily than face-to-face contact because the force required to achieve contact is smaller as shown in Fig. 17. If an external force is required to cause edge-toface contact, flocculation observed in clay samples prepared from slurry should therefore be controlled by the process of mixing.

Once in contact, the edge-to-face is 'soldered' by the van der Waals attraction. However, the interaction at the edge-to-face still remains controlled by the negative charge of the faces of the two particles.

If temperature increases, the double layer repulsion between the two orthogonal particles increases (Fig. 14) and this weakens the contact in the sense that the net attraction decreases. In turn, this makes the contact more prone to collapse.

As discussed by Casarella et al. (2020), this can explain micromechanically the plastic volumetric collapse of normally consolidated clays upon heating. Normally consolidated clays are characterised by a significant amount of edge-to-face contacts (Pedrotti and Tarantino 2018). An increase in temperature increase the repulsion forces significantly (30\% for the case examined in Fig. 12) and this weakens the net attraction predisposing the edge-to-face contact to disengage with consequent plastic deformation.

\section{Conclusions}

The paper has presented an analysis of elementary mechanisms of clay particle interactions with the aim of gaining an insight into the behaviour of clay and advance the process of defining suitable contact laws to be implemented into DEM formulations. Two elementary schemes were analysed theoretically, particles in parallel (face-to-face) configuration and particles in orthogonal (edge-to-face) configuration respectively.

The case of particles in parallel configuration was analysed by considering the cases 'with electrolytes' and 'without' electrolytes. To account for the presence of electrolytes, the Gouy-Champan equations for the diffuse double layers were solved for the case of constant surface density charge. This allowed deriving the interaction pressure using basic concepts of electrostatics.

The response with or without electrolytes appears to be similar qualitatively in the sense that the repulsion interaction pressure tends to a finite value as the inter-particle distance 
approaches zero. This implies that face-to-face contact can be achieved as soon as the external pressure exceeds the maximum repulsion pressure that can be deployed by the particles. Van der Waals forces do not need to be invoked to justify face-to-face contact but play a role in the compression mechanisms as they prevent the particles to detach once the external pressure is relieved.

When particles are in orthogonal configuration, the interaction force remains repulsive even if the edge is charged positively and points to the surface charged negatively. An external force (e.g. Brownian motion) is still required to generate an edge-to-face contact. However, the more positive is the charge of the edge, the higher is the depletion of the repulsion, which facilitates the establishment of the edge-to-face contact.

\section{Acknowledgements}

This work was funded by the 'International Research Centre for Clay Micromechanics' (www.irccm.net) and the ANR project GEO2 (ANR-19-CE05-0003-01). The laboratory 3SR is part of the LabEx Tec 21 (Investissement d'avenir - grant agreement n. ANR11-LABX-0030) and by

\section{References}

Bolland, M.D.A., Posner, A.M., Quirk, J.P., (1976). Surface charge on kaolinites in aqueous suspension. Australian Journal of Soil Research 14, 197-216.

Casarella, A. Pedrotti, M., Tarantino, A., and Di Donna, A. (2020). A critical review of the effect of temperature on clay inter-particle forces and its effect on macroscopic thermal behaviour of clay. 16th International Conference of IACMAG, Torino, Italy, May 2021.

Chen, J., Anandarajah, A. \& Inyang, H. (2000). Pore fluid properties and compressibility of kaolinite. J. Geotech. Geoenviron. Engng 126, No. 9, 798-807.

De Carvalho Faria Lima Lopes, B. Porfírio Cordão Neto, M. And Tarantino A. (submitted). An experimental and theoretical investigation into the micromechanics of plastic deformation of clays: the role of attractive forces. Submitted for publication.

Di Donna, A. \& Laloui, L. (2013). Energy Geostructures: Innovation in Underground Engineering. 10.1002/9781118761809.

Gupta, V.; Miller, J. D. (2010). Surface force measurements at the basal planes of ordered kaolinite particles. J. Colloid Interface Sci., 344(2): 362-371.

Khawmee, K., Suddhiprakarn. A, Kheoruenromne. I, Singh. B. (2013). Surface charge properties of kaolinite from Thai soils. Geoderma 192 (2013) 120-131

Kumar N, Andersson, M.P., van den Ende, H.T.M., Mugele, F.G. Igor Sîretanu (2017). Probing the Surface Charge on the Basal Planes of Kaolinite Particles with High-Resolution Atomic Force Microscopy. Langmuir, 33, 14226-14237

Liu, J.; Sandaklie-Nikolova, L.; Wang, X.; Miller, J. D. (2014). Surface force measurements at kaolinite edge surfaces using atomic force microscopy. J. Colloid Interface Sci., 420, 35-40. 
Mitchell, J. \& Soga, K. 2005. Fundamentals of Soil Behavior, Jon Wiley and Sons Inc. Hoboken, NJ.

Pagano, A.G., Magnanimo,V., Weinhart, T., and Tarantino, A. (2020). Exploring the micromechanics of non-active clays via virtual DEM experiments. Géotechnique, 70(4): 303-316.

Pedrotti, M. and Tarantino, A. (2018). An experimental investigation into the micromechanics of non-active clays. Géotechnique, 68(8): 666-683.

Roscoe, K.H. and Schofield, A.N. (1963). Mechanical behaviour of an idealised. 'wet clay'. Proc. 2nd European Conf. on Soil Mechanics and Foundation Engineering, Wiesbaden, 1 ,47-54.

Schroth, B.K., Sposito, G. (1997). Surface charge properties of kaolinite. Clays and Clay Minerals 45 (1), 85-91.

Van Olphen, H. 1977. Clay Colloid Chemistry: For Clay Technologists, Geologists and Soil Scientists. John Wiley.

Verwey, E. J. W.; Overbeek, J. Th. G. (1948). Theory of the stability of lyophobic colloids. Amsterdam: Elsevier.

Wahid, A. S., Gajo, A. \& Di Maggio, R. (2011). Chemo-mechanical effects in kaolinite. Part 1: prepared samples. Géotechnique 61, No. 6, 439-447, http://dx.doi. org/10.1680/geot.8.P.067.

Wang, Y. H. \& Siu, W. K. (2006). Structure characteristics and mechanical properties of kaolinite soils. I. Surface charges and structural characterizations. Can. Geotech. J. 43, No. 6, 587-600. 